\title{
Os Pacientes Naïve Infectados pelo HIV Apresentam Disfunção Endotelial Concomitante com a Diminuição de Anticorpos Naturais contra Definidos Autoantígenos Derivados da Apolipoproteína B
}

\author{
HIV-Infected Naïve Patients Exhibit Endothelial Dysfunction Concomitant with Decreased Natural Antibodies \\ Against Defined Apolipoprotein B Autoantigens
}

\author{
Henrique Andrade R. Fonseca, ${ }^{1,2}$ Magnus Gidlund, ${ }^{2}$ Viviane Rodrigues Sant'Anna, ${ }^{1,2}$ Esteferson Rodrigues \\ Fernandes, ${ }^{1,2}$ Francisco A. H. Fonseca, ${ }^{100}$ Maria Cristina Izar ${ }^{10}$ \\ Universidade Federal de São Paulo Escola Paulista de Medicina, ${ }^{1}$ São Paulo, SP - Brasil \\ Universidade de Sao Paulo, ${ }^{2}$ São Paulo, SP - Brasil
}

Fundamento: Fatores de risco definidos para HIV e tradicionais podem estar associados a um aumento de eventos cardiovasculares. Estudos recentes sugerem que a resposta imune humoral à LDL modificada pode estar associada ao processo de aterosclerose.

Objetivos: Avaliar a presença de anti-LDL oxidada e de peptídeos derivados da Apolipoproteína B no sangue, bem como sua associação à função endotelial na infecção por HIV.

Métodos: Este estudo incluiu consecutivamente sujeitos com idade, sexo e dados demográficos correspondentes em dois grupos: (1) indivíduos infectados com HIV e naïve para terapia antiviral e (2) indivíduos não infectados. A aterosclerose subclínica foi avaliada pela espessura íntima-média, utilizando-se a ultrassonografia das artérias carótidas. A função endotelial foi determinada pela dilatação mediada por fluxo (DMF) da artéria braquial por ultrassonografia. Os níveis de autoanticorpos (IgM, $\operatorname{lgG}$ ) de lipoproteínas de baixa densidade antioxidadas (LDL-ox), fragmentos de peptídeos antiapolipoproteína B (peptídeos ApoB-D e 0033G-Cys), e citocina foram avaliados por meio de ELISA.

Resultados: Os resultados deste estudo não mostraram diferenças na aterosclerose subclínica entre os grupos. Entretanto, os sujeitos infectados com HIV apresentaram uma DMF mais baixa, em comparação com os sujeitos não infectados. Portanto, os sujeitos infectados com HIV apresentaram níveis mais altos de citocinas inflamatórias, títulos de IgG anti-LDL-ox, e IgG anti-ApoB-D. Em contraste, títulos

Correspondência: Henrique Andrade Fonseca •

Universidade Federal de São Paulo Escola Paulista de Medicina - Rua Loefgreen, 1350. CEP 04023-062, Vila Clementino, São Paulo, SP - Brasil E-mail: har.fonseca@yahoo.com.br

Artigo recebido em 26/01/2020, revisado em 23/04/2020, aceito em $16 / 06 / 2020$

DOI: https://doi.org/10.36660/abc.20200062 de IgM anti-ApoB-D foram mais baixos em indivíduos infectados com HIV e associados a funções endoteliais diminuídas.

Conclusões: Os resultados deste estudo mostram que a infecção por HIV, em sujeitos naïve, está associada à disfunção endotelial e à diminuição de anticorpos naturais para antígenos Apo-B.

\section{Introdução}

Doenças cardiovasculares são mais prevalentes em indivíduos infectados por HIV, em comparação com indivíduos não infectados. ${ }^{1}$ A disfunção endotelial (DE) é o evento iniciador da formação de placas, associado à inflamação do espaço subendotelial causada pela oxidação da proteína de baixa densidade (LDL). ${ }^{2,3}$ A detecção de oxidação de LDL pode ser um marcador do processo e/ou do avanço da aterosclerose. ${ }^{4}$

Para superar alguns dos obstáculos relacionados à falta de mais epítopos restritos do que os expressos em um processo de oxidação artificial (cobre, ferro e outros) para gerar a LDL oxidada (LDL-ox), foi determinada a resposta autoimune a peptídeos da Apolipoproteína B (ApoB) derivada de uma partícula de LDL. Estudos anteriores demonstraram que anticorpos contra um peptídeo específico (ApoB-D) podem ser considerados um marcador de ativação inflamatória. ${ }^{5-7}$ Entretanto, não foi demonstrado que infecções crônicas podem modular os autoanticorpos (AAC) em autoantígenos, especialmente na condição de deficiência do sistema imune.

\section{Materiais e métodos}

\section{Sujeitos}

Este trabalho conduziu um estudo piloto casocontrole transversal que incluiu prospectivamente 40 sujeitos infectados por HIV, naïve para terapia antirretroviral altamente ativa (HAART, do inglês highly active antiretroviral therapy), de ambos os sexos. Cinquenta e três sujeitos não infectados com HIV (controle) foram recrutados nas mesmas comunidades, utilizando-se os mesmos anúncios e fatores de risco cardiovasculares. Depois de fazer a coleta de sangue e avaliações clínicas, 
os pacientes infectados com HIV que iniciaram a HAART aderiram à medicação prescrita.

\section{Lipídios e análise bioquímica}

O colesterol total sérico, o colesterol de lipoproteína de alta densidade (HDL-C), e os triglicérides foram determinados enzimaticamente (Opera Bayer, Leverkusen, Alemanha), com o colesterol de lipoproteína de baixa densidade (LDL-C) estimado pela equação Friedewald quando os triglicérides eram $<400 \mathrm{mg} / \mathrm{dl}^{8}{ }^{8} \mathrm{~A}$ glicose foi avaliada pelo método enzimático.

\section{Função endotelial e espessura íntima-média da carótida}

Testes de ultrassom foram realizados para avaliar a aterosclerose subclínica por meio da espessura íntimamédia da carótida (EIMC) ${ }^{9}$ e avaliação de vasorreatividade da dilatação mediada por fluxo (DMF) dependente do endotélio da artéria braquial. ${ }^{10}$

Brevemente, os pacientes tiveram que fazer jejum e não consumir nitratos e álcool, e não fazer uso de medicamentos vasoativos 24 horas antes dos exames. Depois de um repouso de 15-20 minutos, a artéria braquial da fossa antecubital direita foi visualizada, utilizando-se um transdutor linear com frequência de até $11 \mathrm{MHz}$, com monitoramento simultâneo por eletrocardiograma (ECG). As imagens foram obtidas pelo sistema de ultrassom HP SONOS 5500 (Hewlett Packard, Palo Alto, EUA). Quanto foi obtida a imagem ideal da artéria, o diâmetro do vaso de linha de base foi medido. Foi induzida a hiperemia reativa inflando-se o manguito até $200 \mathrm{mmHg}$, ou pelo menos 50 mmHg acima da PAS, no antebraço distal por 5 minutos e, em seguida, esvaziando-se o manguito. Imagens diastólicas finais foram obtidas no momento do surgimento do complexo QRS no ECG. Essas imagens foram capturadas na linha de base e um minuto após o esvaziamento da braçadeira. A mudança de porcentagem do diâmetro da linha de base até o valor detectado durante a hiperemia reativa foi calculada para determinar a DMF. As medidas de DMF e a EIMC foram avaliadas por um ultrassonografista experiente de maneira cega. As variabilidades de resultados do próprio sonógrafo e entre sonógrafos foram menores que $1 \%$ e $2 \%$, respectivamente.

\section{Citocinas e células T CD4}

As concentrações de citocina foram testadas utilizandose kits ELISA disponíveis comercialmente. A carga viral plasmática e as contagens de células T CD4 foram determinadas para os sujeitos infectados com HIV. O nadir de células T CD4+ foi definido como o valor mais baixo registrado e confirmado em laboratório.

\section{Isolamento e síntese de autoantígeno}

A partícula de LDL foi obtida do plasma total após a centrifugação ( $\left.1.000 \mathrm{~g} ; 4{ }^{\circ} \mathrm{C} ; 15 \mathrm{~min}\right)$ e suplementada com benzamidina $(2 \mathrm{mM})$, gentamicina $(0,5 \%)$, cloranfenicol $(0,25 \%)$, fluoreto de fenilmetilsulfonil (PMSF) $(0,5 \mathrm{mM})$, e aprotinina $(0,1$ unidade/mL). Partículas de lipoproteína de baixa densidade $(1,006<\mathrm{d}<1,063 \mathrm{mg} / \mathrm{mL})$ foram isoladas por ultracentrifugação sequencial (100.000 g; 4 ${ }^{\circ} \mathrm{C}$ ), utilizando-se um rotor (70 Ti, ângulo fixo; Beckman Coulter, EUA) e uma ultracentrífuga (Hitachi, Japão). A partícula de LDL foi oxidada por cobre e utilizada como autoantígeno para avaliar os títulos de anticorpos. ${ }^{11}$ Os peptídeos de Apolipoproteína B (peptídeos ApoB) usados neste estudo foram compostos de dois fragmentos sintéticos: ApoB-D (ApoB-D, que é um fragmento de peptídeo ApoB com uma sequência de 22 aminoácidos derivados do domínio 3 da sequência da Apolipoproteína $B$ na terceira porção conservada para digestões de tripsina), ${ }^{12}$ e o peptídeo-0033G-Cys (peptídeo-0033GCys, que é um fragmento de peptídeo com uma sequência de 21 aminoácidos derivados do domínio 3 da sequência da Apolipoproteína B na primeira porção conservada para digestão de tripsina). ${ }^{12}$

\section{Determinações de autoanticorpos}

A quantificação da LDL-ox e dos autoanticorpos (AAC) derivados do peptídeo ApoB foi avaliada no plasma total por ELISA, conforme descrito anteriormente. ${ }^{13,14}$ Microplacas de 96 poços (Microplates 8096, CostarEUA) foram cobertas com $10 \mu \mathrm{g} / \mathrm{mL}$ do peptídeo ApoB-D ou 0033G-Cys em tampão carbonato/bicarbonato $(0,1$ $\mathrm{mol} / \mathrm{l} ; \mathrm{pH} 9,6)$, que foi deixado em sensibilização durante a noite a $4^{\circ} \mathrm{C}$. Depois de três ciclos de lavagem com solução salina tamponada com fosfato (PBS, pH 7,4) mais Polissorbato-20 (0,05\%), a placa foi bloqueada com gelatina (3\%; temperatura ambiente; $24 \mathrm{~h}$ ). Amostras de plasma dos pacientes (50 $\mu \mathrm{l} /$ poço, 1:400 em tampão fosfatado, PBS, pH 7,4) foram acrescentadas às placas por 2 horas em temperatura ambiente. Em seguida, três ciclos adicionais de lavagem foram realizados, e foram acrescentados anticorpos conjugados com peroxidase horseradish de IgG secundário (anticorpo de cabra anti-lgG de humano, 0,1 $\mu \mathrm{g} / \mathrm{ml}, \mathrm{KPL}$, Kirkegaard \& Perry Laboratories, Gaithersburg, Maryland, EUA) ou IgM (anticorpo de cabra anti-IgM de humano purificado, $10 \mu \mathrm{g} / \mathrm{ml}$, KPL, Kirkegaard \& Perry Laboratories, Gaithersburg, Maryland, EUA), para avaliar os títulos de AAC de peptídeos anti-ApoB-D ou anti-0033G-Cys. Depois da incubação (1 hora), as placas foram lavadas (três ciclos), e foram acrescentados 3,3,5,5-tetrametilbenzidina (6,5\% in dimetilsulfóxido; Sigma, St Louis, MO) e $\mathrm{H}_{2} \mathrm{O}_{2}$ (Sigma) diluídos em tampão fosfato-citrato $(0,1 \mathrm{~mol} / \mathrm{l} ; 250 \mu \mathrm{l} ; \mathrm{pH}$ $5,5)$ em temperatura ambiente, como enzima-substratos. A reação foi interrompida acrescentando-se $\mathrm{H}_{2} \mathrm{SO}_{4}(2 \mathrm{~mol} / \mathrm{l})$. A densidade ótica (DO) das amostras foi medida em 450 $\mathrm{nm}$. Títulos de autoanticorpos (AAC) foram expressos como índice de reatividade (IR), calculado como IR $=\left(\mathrm{DO}_{\text {amostra }}-\right.$ $\mathrm{DO} \mathrm{amostra} \mathrm{em} \mathrm{branco}) /\left(\mathrm{DO}_{\mathrm{lgG} \text { ou IgM }}-\mathrm{DO} \mathrm{lgG} \mathrm{ou} \mathrm{IgM} \mathrm{em} \mathrm{branco}_{\text {amostra }}\right)$ em que os anticorpos IgG ou IgM foram usados como controles. O coeficiente de variação intraensaios foi de 5,4\% e o intraensaios foi de $2,0 \%$.

Foram realizados títulos de AAC Anti-LDL-ox similares para o ensaio com peptídeo de Apolipoproteína B, utilizando-se, contudo, microplacas de noventa e seis poços cobertos com 7,5 $\mu \mathrm{g} / \mathrm{ml}$ de LDL-ox. ${ }^{13}$ Os anticorpos totais foram determinados em plasma total pelo método ELISA. 
As amostras foram executadas triplicadas e a variação entre os triplos não ultrapassou 5\% da média.

\section{Ética}

O estudo foi aprovado pelo comitê de ética institucional da Universidade Federal de São Paulo e da Universidade de São Paulo (FO. 99/2009), e todos os participantes assinaram termos de consentimento informado antes do início do protocolo.

\section{Análise estatística}

As análises estatísticas foram realizadas utilizando-se o pacote de software SPSS 17.0 (Statistical Package for Social Science, SPSS Inc., Chicago, IL, EUA). As variáveis categóricas foram comparadas por meio do teste qui-quadrado de Pearson. A distribuição de normalidade foi avaliada pelo teste de Kolmogorov-Smirnov. As análises entre grupos foram testadas por teste $t$ ou teste Mann-Whitney. A interação entre a função endotelial e outras variáveis foram testadas com os testes de Pearson ou Spearman. Variáveis cuja interação foi identificada como significativa foram testadas com análise de regressão linear múltipla stepwise, com a função endotelial como variável dependente. Foi usado um nível de significância de 5\% para todos os testes.

\section{Resultados}

Os parâmetros clínicos e demográficos são apresentados na Tabela 1. Não houve diferença de EIMC entre sujeitos infectados com HIV e não infectados. A função endotelial foi diminuída em sujeitos infectados com HIV ( $p=0,040)$. (Tabela 1).

Como esperado, sujeitos infectados com HIV tinham níveis de marcador inflamatório significativamente mais altos do que indivíduos não infectados. Entretanto a citocina anti-inflamatória IL-10 não variou entre os grupos (Tabela 1).

Os títulos de AAC IgG e IgM totais séricos não variaram entre sujeitos infectados e não infectados com HIV (Tabela 1). A Figura 1 demonstrou que os títulos de AAC de IgG anti-LDL-ox eram mais altos em sujeitos infectados por HIV $(p<0.001)$. Entretanto, os títulos de AAC de IgM anti-LDLox não variaram entre sujeitos infectados e não infectados com HIV. Os sujeitos infectados com HIV tiveram títulos mais altos de AAC de IgG anti-ApoB-D $(p<0,001)$ e títulos mais baixos de IgM anti-ApoB-D em comparação com sujeitos não infectados $(p=0,040)$. Nenhuma diferença foi encontrada entre os grupos em relação aos AAC de antipeptídeo-0033G-Cys.

O presente estudo demonstrou que, em sujeitos infectados com HIV, a função endotelial foi associada aos títulos IgM anti-ApoB-D AAC $[\beta=10,75 ; p=0,015]$ (Tabela 2). O modelo de regressão stepwise, incluindo fatores de risco cardiovascular tradicionais, marcadores relacionados ao HIV, e respostas imunes, mostrou que AAC IgM anti-ApoB-D estavam associados à função endotelial $[\beta=7,28 ; p=0,002]$. Não foram observadas associações entre IgG anti-ApoB-D e a função endotelial. Em relação à aterosclerose subclínica, as medidas de EIMC não foram associadas à resposta humoral para ambos os peptídeos.

\section{Discussão}

O presente estudo mostrou que, em sujeitos infectados por HIV, naïve de terapia antirretroviral, uma função endotelial reduzida acompanha uma modulação distinta em AAC contra fragmentos de peptídeos ApoB, em comparação com sujeitos não infectados, independentemente dos títulos de AAC com totais séricos.

Dados relacionados a imunidade humoral de peptídeos ApoB sugerem que sua presença está associada ao avanço da doença aterosclerótica, como parte de uma resposta autoimune. 5,14 Entretanto, esses AAC podem participar de eliminação de produtos pró-aterogênicos gerados a partir da oxidação de partículas LDL, e da modificação dos ApoB, desempenhando uma função dupla no processo de aterogênese. ${ }^{15,16}$

Este estudo também mostrou que AAC de IgM contra ApoB-D estavam associados à ED, corroborando com estudos anteriores. ${ }^{6,17}$ Nossos achados sugerem que há uma eliminação de autoantígenos ApoB por anticorpos naturais, sugerindo que eles possam estar envolvidos em reparos vasculares após um processo de lesão, ${ }^{18}$ entretanto, os efeitos da infecção por HIV da DMF podem ser atribuídos a um estágio distinto da doença e às diferentes terapias medicamentosas adotadas. ${ }^{19}$

Estudos de coorte e meta-análise demonstraram que a EIMC é mais alta em pacientes infectados com HIV, em comparação com não infectados. ${ }^{20}$ Acreditamos que o momento da infecção em nosso estudo não foi suficiente para promover modificações ateroscleróticas na carótida, detectadas por exame de ultrassom.

Os resultados do presente estudo sugerem que autoanticorpos para peptídeos definidos-ApoB podem ser um marcador de disfunção endotelial, ou mesmo de uma resposta inflamatória elevada, porém não de aterosclerose da carótida em pacientes infectados com HIV. Estudos clínicos coorte em pacientes submetidos a HAART merecem investigação adicional para confirmar esses resultados preliminares.

O desenho transversal e a falta de um grupo que estivesse recebendo tratamento antirretroviral para permitir comparações dos efeitos das drogas HAART na função endotelial e na aterosclerose subclínica são limitações. Nenhuma diferença significativa foi encontrada entre os sexos, o que pode ser justificado pelo pequeno número de sujeitos incluído neste estudo. Estudos adicionais, incluindo um número maior de pacientes, são necessários para confirmar nossos achados em relação a sexo, infecção e função endotelial. Para fins de ajuste, os efeitos de fatores de risco cardiovascular diferentes e marcadores de infecção foram avaliados como uma possível explicação para a resposta imune natural observada, associada à função vascular.

\section{Conclusão}

Os achados deste estudo sugerem que a imunidade natural a antígenos ApoB está associada à DE. Mais estudos prospectivos são necessários para a avaliação de parâmetros 
Tabela 1 - Características dos sujeitos infectados com HIV naïve e não infectados

\begin{tabular}{|c|c|c|c|c|}
\hline Variáveis & $\begin{array}{c}\text { Geral } \\
\text { (93) }\end{array}$ & $\begin{array}{c}\text { HIV- } \\
\text { (Controle) } \\
\text { (53) }\end{array}$ & $\begin{array}{l}\text { HIV+ } \\
\text { (Naî̀ve) } \\
\text { (40) }\end{array}$ & p-valores \\
\hline \multicolumn{5}{|l|}{ Parâmetros clínicos } \\
\hline Sexo (masculino/feminino) & $63 / 30$ & $32 / 21$ & $31 / 9$ & 0,110 \\
\hline Idade (anos) & $32(1,0)$ & $32(1,7)$ & $32(1,3)$ & 0,746 \\
\hline Circunferência abdominal (cm) & $88(83-97)$ & $89,5(76,5-100)$ & $97(83-96)$ & 0,668 \\
\hline Índice de Massa Corporal ( $\left.\mathrm{kg} / \mathrm{m}^{2}\right)$ & $24,8(23-28)$ & $25,5(21,5-28,5)$ & $25,2(23,5-28)$ & 0,586 \\
\hline Fumantes (\%) & 11 & 6 & 5 & 0,951 \\
\hline Pressão sanguínea sistólica (mmHg) & $120(110-120)$ & $120(110-120)$ & $120(110-120)$ & 0,631 \\
\hline Pressão sanguínea diastólica (mmHg) & $80(70-80)$ & $80(70-80)$ & $80(70-80)$ & 0,441 \\
\hline \multicolumn{5}{|l|}{ Análise bioquímica } \\
\hline Colesterol total $(\mathrm{mg} / \mathrm{dL}) \mathrm{z}$ & $165(139-185)$ & $166(144-191)$ & $150(124-176)$ & 0,028 \\
\hline LDL-c (mg/dL) & $98(69-115)$ & $103(77-119)$ & $91(66-113)$ & 0,095 \\
\hline $\mathrm{HDL}-\mathrm{c}(\mathrm{mg} / \mathrm{dL})$ & $46(37-65)$ & $47(40-57)$ & $36(30-46)$ & 0,008 \\
\hline Triglicérides (mg/dL) & $91(52-122)$ & $88(67-131)$ & $113(73-131)$ & 0,285 \\
\hline Glicose (mg/dL) & $90(86-94)$ & $90(86-94)$ & $92(86-96)$ & 0,425 \\
\hline \multicolumn{5}{|l|}{ Parâmetros de infecção por HIV } \\
\hline Tempo de infecção (anos) & - & - & $3(1-6)$ & N.A \\
\hline Contagem de células CD4 (células/ $\mu \mathrm{L}$ ) & - & - & $447(366-590)$ & N.A \\
\hline Nadir CD4 (células/ $\mu \mathrm{L}$ ) & - & - & $402(356-537)$ & N.A \\
\hline Carga viral de HIV (cópias de RNA/ $\mu \mathrm{L}$ ) & - & - & $2623(485-26225)$ & N.A \\
\hline Coinfecção por HBV & 0 & 0 & 4 & N.A \\
\hline Coinfecção por HCV & 0 & 0 & 3 & N.A \\
\hline \multicolumn{5}{|l|}{ Terapia em uso } \\
\hline Anti-hipertensivos (indivíduos, N) & 4 & 3 & 1 & N.A \\
\hline Estatinas (indivíduos, N) & 0 & 0 & 0 & N.A \\
\hline Drogas neurológicas (indivíduos, N) & 3 & 2 & 1 & N.A \\
\hline \multicolumn{5}{|l|}{ Marcadores inflamatórios } \\
\hline hs-CRP (mg/L) & $1,20(0,30-1,92)$ & $0,51(0,20-1,87)$ & $1,48(0,82-3,30)$ & 0,017 \\
\hline IFN-y (pg/dL) & $2,84(0,90-6,85)$ & $1,43(0,87-4,10)$ & $3,89(1,30-8,85)$ & 0,021 \\
\hline TNF- $\alpha(p g / d L)$ & $6,66(5,58-7,31)$ & $6,02(5,51-6,94)$ & $6,90(6,54-7,63)$ & 0,020 \\
\hline $\mathrm{IL}-6$ (pg/dL) & $1,54(1,37-1,80)$ & $1,54(1,36-1,63)$ & $1,50(1,37-1,95)$ & 0,028 \\
\hline IL-8 (pg/dL) & $3,13(2,50-4,60)$ & $2,80(2,20-4,40)$ & $3,65(2,70-5,50)$ & 0,050 \\
\hline IL-10 (pg/dL) & $1,75(0,39-1,97)$ & $1,79(0,80-1,98)$ & $0,87(0,36-1,94)$ & 0,088 \\
\hline \multicolumn{5}{|l|}{ Anticorpos totais } \\
\hline Total sérico de IgG (IR) & $1,33(1,19-1,38)$ & $1,34(1,20-1,38)$ & $1,33(1,18-1,37)$ & 0,877 \\
\hline Total sérico de IgM (IR) & $0,69(0,55-0,84)$ & $0,67(0,49-0,82)$ & $0,3(0.58-0.86)$ & 0,310 \\
\hline \multicolumn{5}{|l|}{ Aterosclerose subclínica } \\
\hline Espessura íntima-média (mm) & $0,67(0,57-0,68)$ & $0,67(0,56-0,68)$ & $0,67(0,57-0,68)$ & 0,971 \\
\hline \multicolumn{5}{|l|}{ Função endotelial } \\
\hline Dilatação mediada por fluxo (\%) & $11,6(1,4)$ & $13,7(2,4)$ & $9,3(1,2)$ & 0,040 \\
\hline
\end{tabular}

HBV: virus da hepatite B; HCV: virus da hepatite C; N.A: não se aplica; IR: índice de reatividade. 


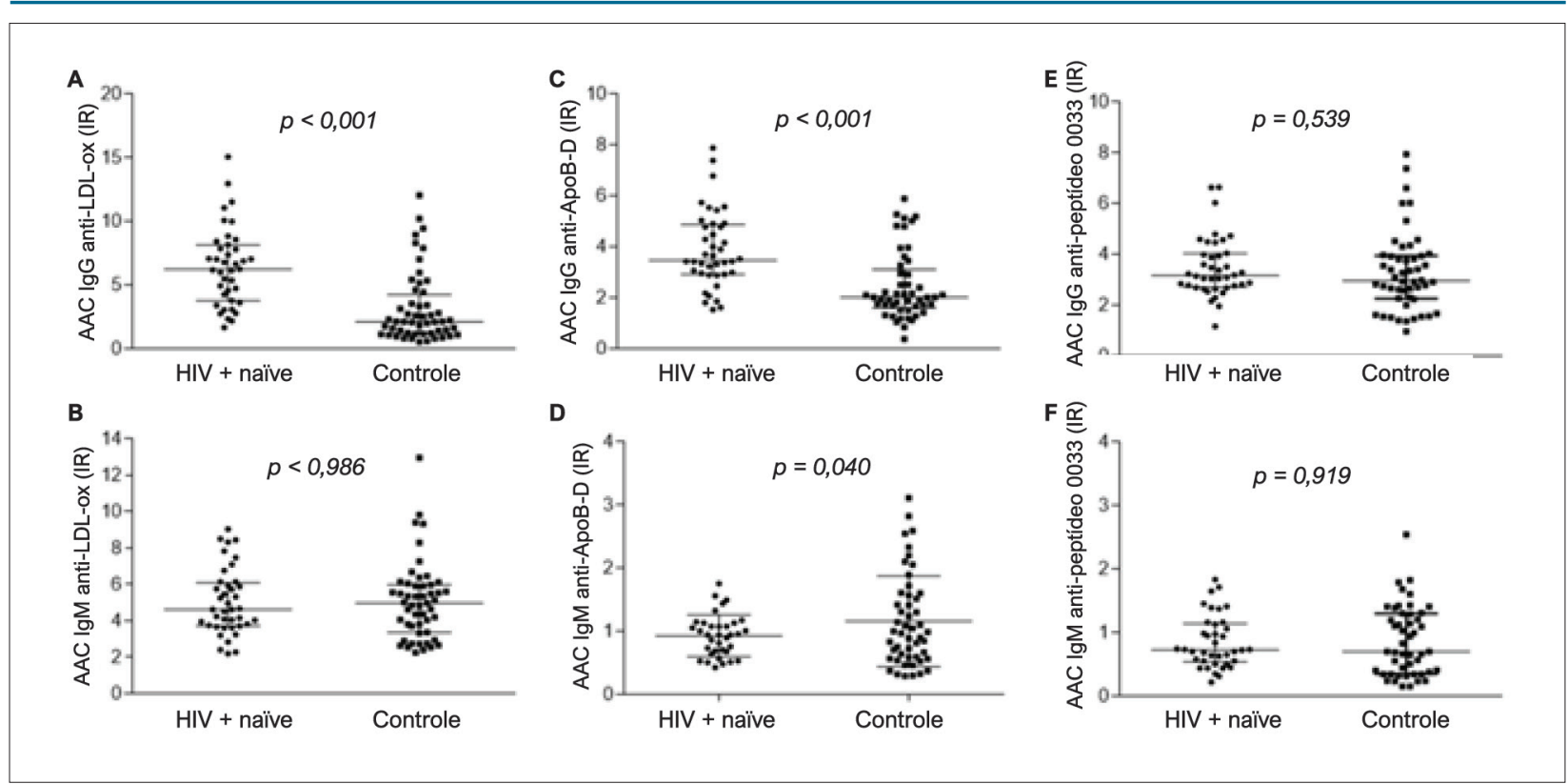

Figura 1 - Resposta humoral a LDL oxidada, peptideo ApoB-D e peptideo 0033 em pacientes infectados com HIV e controles não infectados. (A) Autoanticorpos (AAC) IgG anti-LDL-ox; (B) AAC IgM anti-LDL-ox; (C) AAC IgG peptídeo anti-ApoB-D; (D) AAC IgM anti-ApoB-D. (E) AAC IgG peptídeos anti-0033; (F) IgM peptídeos anti-0033. Diferenças significativas entre os grupos foram calculadas pelo teste de Mann-Whitney.

Tabela 2 - Análise univariada ajustada de possíveis fatores de risco associados à função endotelial em sujeitos infectados com HIV

\begin{tabular}{lcc}
\hline \multirow{2}{*}{ Variáveis } & & \\
\cline { 2 - 3 } & \multicolumn{1}{c}{$\boldsymbol{\beta}$} & $\mathbf{p}$-valores \\
\hline Idade (anos) & $-0,187$ & 0,350 \\
\hline Circunferência abdominal & $-0,049$ & 0,708 \\
\hline IgM peptídeo anti-ApoB-D & 10,754 & 0,015 \\
\hline IgG peptídeo anti-ApoB-D & 0,597 & 0,351 \\
\hline Nadir CD4 & 0,007 & 0,135 \\
\hline CD4 atual & $-0,010$ & 0,126 \\
\hline Carga viral registrada & 0,413 & 0,786 \\
\hline Tempo de infecção & $-0,215$ & 0,718 \\
\hline
\end{tabular}

0 coeficiente $\beta$ representa as alterações na porcentagem de dilatação mediada por fluxo nas variáveis preditoras. Foram feitos ajustes para hipertensão, tabagismo atual, e dislipidemia. IC: Intervalo de confiança.

imunológicos de HIV em resposta autoimune e seus efeitos na função vascular.

\section{Contribuição dos autores}

Concepção e desenho da pesquisa e Revisão crítica do manuscrito quanto ao conteúdo intelectual importante: Fonseca HA, Gidlund M, Fonseca FAH, Izar MC; Obtenção de dados: Fonseca HA, Fernandes ER; Análise e interpretação dos dados: Fonseca HA, Gidlund M, Sant'Anna VR, Fernandes ER; Análise estatística: Fonseca HA, Sant'Anna VR; Obtenção de financiamento: Fonseca HA, Fonseca FAH; Redação do manuscrito: Fonseca HA, Gidlund M, Sant'Anna VR, Izar MC.

\section{Potencial conflito de interesses}

Os autores declaram não haver conflito de interesses pertinentes.

\section{Fontes de financiamento}

O presente estudo não contou com fontes de financiamento externas.

\section{Vinculação acadêmica}

Não há vinculação deste estudo a programas de pósgraduação. 


\section{Errata}

Na Comunicação Breve "Pacientes Naïve Infectados por HIV Apresentam Disfunção Concomitante com Diminuição de Anticorpos Naturais contra Autoantígenos Derivados da Apolipoproteína B Definidos", com número de DOI: https://doi.org/10.36660/abc.20200062, publicado no periódico Arquivos Brasileiros de Cardiologia, 116(4):844-849, na página 844, corrigir o título do artigo em português "Pacientes Naïve Infectados por HIV Apresentam Disfunção Concomitante com Diminuição de Anticorpos Naturais contra Autoantígenos Derivados da Apolipoproteína B Definidos" para: "Os Pacientes Naïve Infectados pelo HIV Apresentam Disfunção Endotelial Concomitante com a Diminuição de Anticorpos Naturais contra Definidos Autoantígenos Derivados da Apolipoproteína B".

\section{Referências}

1. Feinstein MJ, Hsue PY, Benjamin LA, Bloomfield GS, Currier JS, Freiberg MS, et al. Characteristics, prevention, and management of cardiovascular disease in people living with HIV: a scientific statement from the American Heart Association. Circulation. 2019;140(2):e98-124.

2. Kearns A, Gordon J, Burdo TH, Qin X. HIV-1-Associated atherosclerosis: unraveling the missing link. J Am Coll Cardiol. 2017;69(25):3084-98.

3. Stein JH, Currier JS, Hsue PY. Arterial disease in patients with human immunodeficiency virus infection: what has imaging taught us? JACC Cardiovasc Imaging. 2014;7(5):515-25.

4. PocockMO, Dorrell L, Cicconi P. Pathophysiology of ischaemic heart disease. Curr Opin HIV AIDS. 2017;12(6):548-53.

5. Fonseca HAR, Fonseca FA, Monteiro AM, Bianco HT, Boschcov P, Brandão $\mathrm{SA}$, et al. Obesity modulates the immune response to oxidized LDL in hypertensive patients. Cell Biochem Biophys. 2013;67(3):1451-60.

6. Fonseca HAR, Fonseca FA, Lins LC, Monteiro AM, Bianco HT, Brandão $\mathrm{SA}$, et al. Antihypertensive therapy increases natural immunity response in hypertensive patients. Life Sci. 2015 Dec 15;143:124-30.

7. Svensjö, Boschcov P, Ketelhuth DF, Jancar S, Gidlunf M. Increased microvascular permeability in the hamster cheek pouch induced by oxidized low density lipoprotein (oxLDL) and some fragmented apolipoprotein B proteins. Inflamm Res. 2003;52:215-20.

8. Friedewald WT, Levy RI, Fredrickson DS. Estimation of the concentration of low-density lipoprotein cholesterol in plasma, without use of the preparative ultracentrifuge. Clin Chem. 1972;18(6):499-502.

9. Stein JH, Korcarz CE, Hurst RT, Lonn E, Kendall CB, Mohler ER, et al. American Society of Echocardiography Carotid Intima-Media Thickness Task Force. Use of carotid ultrasound to identify subclinical vascular disease and evaluate cardiovascular disease risk: a consensus statement from the American Society of Echocardiography Carotid Intima-Media Thickness Task Force. Endorsed by the Society for Vascular Medicine. J Am Soc Echocardiogr. 2008;21(2):93-111.

10. Corretti MC, Anderson TJ, Benjamin EJ, Celermajer D, Charbonneau F, Creager MA, et al. Guidelines for the ultrasound assessment of endothelialdependent flow-mediated vasodilation of the brachial artery. a report of the International Brachial Artery Reactivity Task Force. J Am Coll Cardiol. 2002;39(2):257-65.
11. Fernvik EC, Ketelhuth DF, Russo M, Gidlund M. The autoantibody repertoire against copper- or macrophage-modified LDL differs in normolipidemics and hypercholesterolemic patients. J Clin Immunol. 2004;24(2):170-6.

12. Yang CY, Gu ZW, Weng SA, Kim TW, Chen SH, Pownall HJ, et al. Structure of apolipoprotein B-100 of human low density lipoproteins. Arteriosclerosis. 1989;9(1):96-108.

13. Fonseca HAR, Fonseca FAH, Monteiro AM, Farias Jr NC, Bianco HT, Brandão $\mathrm{SAB}$, et al. Inflammatory environment and immune responses to oxidized LDL are linked to systolic and diastolic blood pressure levels in hypertensive subjects. Int J Cardiol. 2012;157(1):131-3.

14. Soares SR, Carvalho-Oliveira R, Ramos-Sanchez E, Catazoni S, Silva LFF, Mauad T, et al. Air pollution and antibodies against modified lipoproteins are associated with atherosclerosis and vascular remodeling in hyperlipemic mice. Atherosclerosis. 2009;207(2):368-73.

15. Lopes-Virella MF, Virella G. Pathogenic role of modified LDL antibodies and immune complexes in atherosclerosis. J Atheroscler Thromb. 2013;20(10):743-54.

16. Ronchini KR, Duarte AJ, Casseb JSR, Gidlund M. Cardiovascular complications and increased levels of circulating modified low density lipoprotein in HIV patients and patients with lipodystrophy. Braz J Med Biol Res. 2004;37(1):119-22.

17. Orellana RV, Fonseca HA, Monteiro AM, Ortega KL, Gallottini MH, Gidlund M, et al. Association of autoantibodies anti-OxLDL and markers of inflammation with stage of HIV infection. Int J Cardiol. 2013;168(2):1610-2.

18. Asciutto G, Wigren M, Fredrikson GN, Mattisson IY, Grönberg C, Alm R, et al. Apolipoprotein B-100 antibody interaction with atherosclerotic plaque inflammation and repair processes. Stroke. 2016;47(4):1140-3.

19. Bush KNV, Teel JL, Watts JA, Gore RS, Alvarado G, Harper NL, et al. Association of endothelial dysfunction and antiretroviral therapy in early HIV infection. JAMA Netw Open. 2019;2(10):e1913615.

20. Wohl DA, Arnoczy G, Fichtenbaum CJ, Campbell T, Taiwo B, Hicks C, et al. Comparison of cardiovascular disease risk markers in HIV-infected patients receiving abacavir and tenofovir: the nucleoside inflammation, coagulation and endothelial function (NICE) study. Antivir Ther. 2014;19(2):141-7. 
Edição de Abril de 2021, vol. 116 (4), págs. 844-849

Na Comunicação Breve "Pacientes Naïve Infectados por HIV Apresentam Disfunção Concomitante com Diminuição de Anticorpos Naturais contra Autoantígenos Derivados da Apolipoproteína B Definidos", com número de DOI: https://doi.org/10.36660/abc.20200062, publicado no periódico Arquivos Brasileiros de Cardiologia, 116(4):844-849, na página 844, corrigir o título do artigo em português "Pacientes Naïve Infectados por HIV Apresentam Disfunção Concomitante com Diminuição de Anticorpos Naturais contra Autoantígenos Derivados da Apolipoproteína B Definidos" para: "Os Pacientes Naïve Infectados pelo HIV Apresentam Disfunção Endotelial Concomitante com a Diminuição de Anticorpos Naturais contra Definidos Autoantígenos Derivados da Apolipoproteína B". 